\title{
Correction to: Utilizing the MODIS-derived leaf area index to investigate the impact of vegetation processes on hydrological simulation of macroscale catchment
}

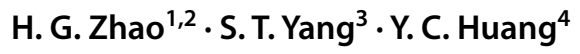

Published online: 13 February 2018

๑) Springer-Verlag GmbH Germany, part of Springer Nature 2018

Correction to: Environmental Earth Sciences (2018) 77:11
https://doi.org/10.1007/s12665-017-7187-3

Unfortunately, the online version of the original article has an error in authors' affiliations.

In the original article, the authors' affiliations were accidentally changed during the page proof corrections. The correct affiliation is given below:

H. G. Zhao ${ }^{1,2} \bullet$ S. T. Yang ${ }^{3} \bullet$ Y. C. Huang ${ }^{4}$

1 Beijing Water Science and Technology Institute, Beijing 100048, China;

2 Institute of Environment and Sustainable Development in Agriculture, Chinese Academy of Agricultural Sciences, 100081 Beijing, China;

3 College of Water Sciences, Beijing Normal University 100875, China;

4 Beijing Institute of Surveying and Mapping, Beijing 100038, China.

The original article can be found online at https://doi.org/10.1007/ s12665-017-7187-3.

H. G. Zhao

zhaohaigen1983@163.com

1 Beijing Water Science and Technology Institute, Beijing 100048, China

2 Institute of Environment and Sustainable Development in Agriculture, Chinese Academy of Agricultural Sciences, Beijing 100081, China

3 College of Water Sciences, Beijing Normal University, Beijing 100875, China

4 Beijing Institute of Surveying and Mapping, Beijing 100038, China 